\title{
Reheating processes after Starobinsky inflation in old-minimal supergravity
}

\author{
Takahiro Terada, ${ }^{a}$ Yuki Watanabe, ${ }^{b}$ Yusuke Yamada ${ }^{c}$ and Jun'ichi Yokoyama ${ }^{b, d}$ \\ ${ }^{a}$ Department of Physics, The University of Tokyo, \\ 7-3-1 Hongo, Tokyo 113-0033, Japan \\ ${ }^{b}$ Research Center for the Early Universe (RESCEU), \\ Graduate School of Science, The University of Tokyo, \\ 7-3-1 Hongo, Tokyo 113-0033, Japan \\ ${ }^{c}$ Department of Physics, Waseda University, \\ 3-4-1 Okubo, Tokyo 169-8555, Japan \\ ${ }^{d}$ Kavli Institute for the Physics and Mathematics of the Universe (Kavli IPMU), \\ WPI, TODIAS, The University of Tokyo, \\ 5-1-5 Kashiwanoha, Chiba 277-8568, Japan \\ E-mail: takahiro@hep-th.phys.s.u-tokyo.ac.jp, \\ watanabe@resceu.s.u-tokyo.ac.jp, yuusuke-yamada@asagi.waseda.jp, \\ yokoyama@resceu.s.u-tokyo.ac.jp
}

ABSTRACT: We study reheating processes and its cosmological consequences in the Starobinsky model embedded in the old-minimal supergravity. First, we consider minimal coupling between the gravity and matter sectors in the higher curvature theory, and transform it to the equivalent standard supergravity coupled to additional matter superfields. We then discuss characteristic decay modes of the inflaton and the reheating temperature $T_{\mathrm{R}}$. Considering a simple model of supersymmetry breaking sector, we estimate gravitino abundance from inflaton decay, and obtain limits on the masses of gravitino and supersymmetry breaking field. We find $T_{\mathrm{R}} \simeq 1.0 \times 10^{9} \mathrm{GeV}$ and the allowed range of gravitino mass as $10^{4} \mathrm{GeV} \lesssim m_{3 / 2} \lesssim 10^{5} \mathrm{GeV}$, assuming anomaly-induced decay into the gauge sector as the dominant decay channel.

KEYwords: Cosmology of Theories beyond the SM, Supergravity Models, Supersymmetry Breaking

ArXiv EPRINT: 1411.6746 


\section{Contents}

1 Introduction 1

2 Starobinsky model embedded in matter-coupled old-minimal supergravity

3 Inflaton decay $\quad \mathbf{7}$

$\begin{array}{lll}3.1 & \text { Two-body decay of } T \text { into scalars, spinors and gauge bosons } & 7\end{array}$

$\begin{array}{lll}3.1 .1 & \text { Decay into scalars } & 7\end{array}$

3.1.2 Decay into spinors $\quad 8$

3.1.3 Anomaly-induced decay into gauge sector 9

$\begin{array}{llr}3.2 & \text { Three-body decay of } T & 9\end{array}$

$\begin{array}{lll}3.3 & \text { Decay of } S & 10\end{array}$

$\begin{array}{ll}3.4 \text { Gravitino production } & 10\end{array}$

$\begin{array}{lll}3.4 .1 & \text { Single gravitino production } & 10\end{array}$

$\begin{array}{ll}\text { 3.4.2 Gravitino pair production } & 11\end{array}$

4 Constraints from gravitino abundance $\quad 13$

$\begin{array}{lll}5 & \text { Summary and discussion } & 17\end{array}$

A Duality transformation of higher derivative SUGRA models 18

\section{Introduction}

The recent observations of cosmic microwave background (CMB) by the WMAP [1] and Planck satellites $[2,3]$ indicate that nature is simple and minimal, providing increasing evidence to favor single field inflationary models.

Among the pioneering [4-10] and newer models of inflation $[12],{ }^{1}$ Starobinsky model $[4$, 5] occupies a unique position, since it does not require any new field to drive inflation which we call the inflaton. Its original version $[4,5]$ is based on a higher curvature action which emerges by incorporating matter loops to the Einstein Hilbert action. Since all the secondorder contributions of curvature tensors that affect the Einstein equation in a conformally flat geometry including the Robertson-Walker spacetime can be adequately described by the square scalar curvature term, currently popular version of Starobinsky model simply consists of linear and second-order terms of the Ricci scalar $R$ [13].

In this model inflation is followed by an oscillatory behavior of the Hubble parameter, which results in gravitational particle production to reheat the Universe. Making use of a conformal transformation, one can also recast the system to the Einstein action containing

\footnotetext{
${ }^{1}$ For a review of inflation see e.g. ref. [11].
} 
a scalar field, dubbed as the scalaron acting as the inflaton, with a specific potential whose overall magnitude is determined by the coefficient of $R^{2}$ term in the original action [14, 15]. In this picture reheating is described by the decay of the scalaron. Various aspects of reheating after $R^{2}$ inflation have been studied in refs. [16-23]. Ref. [19] showed that dark matter and baryon asymmetry are produced at reheating by introducing Majorana neutrinos. Ref. [23] showed that parametric resonance is not strong enough to form long-living localized objects, and thus reheating proceeds through perturbative decay of the inflaton.

The only adjustable parameter of Starobinsky model, namely the coefficient of the curvature square term, can be fixed by the amplitude of curvature perturbation [24, 25]. Its spectral index $n_{s}$ and the tensor-to-scalar ratio $r$ have also been confronted with observations, and interestingly, this oldest inflation model occupies just the central region of their error ellipses [1-3]. This feature was challenged this March by BICEP2 collaboration [26], which claimed to have detected B-mode polarization of CMB corresponding to a value of $r$ much larger than favored by these satellite based observations. It turned out later, however, that the contamination of foreground dust may be so significant that one cannot rule out models with small $r$ yet at all [27-29]. Thus the observational validity of Starobinsky model is still intact.

Needless to say, on the other hand, occupying the central region of the likelihood contour does not necessarily mean the model is the right one, and we should continue our efforts to further clarify features of Starobinsky model in the context of modern high energy theories, in particular, in supersymmetry (SUSY) which reduces the hierarchy problem significantly, naturally realizes gauge coupling unification, and provides cold dark matter candidates.

In the case of Starobinsky model, which is a theory of gravity, SUSY actually means supergravity (SUGRA) [30, 31]. There are two minimal choices for the SUGRA multiplet: the old-minimal [32-34] and new-minimal [35] formulations. These two formulations utilize different SUGRA auxiliary fields, but they coincide on-shell for the standard SUGRA action, whose bosonic part is General Relativity. ${ }^{2}$ This situation changes in the case of higher-derivative SUGRA, including SUGRA versions of Starobinsky model, because these auxiliary fields become propagating degrees of freedom. Embedding of the Starobinsky action into the old-minimal SUGRA was studied at the linearized level in ref. [37] and the non-linear level in ref. [38], where the duality to the standard SUGRA action with additional superfields was also established (in analogy with the bosonic case [14, 15]). Tachyonic instability during the inflationary phase was cured in ref. [39]. In this SUGRA setup, the $R+R^{2}$ action (without higher order terms) emerges from generic $F$ - and $D$-term action (without derivatives and superderivatives) [38, 40] (see also refs. [41, 42]). The limited case, F-term generic action, was rediscovered in ref. [43] and developed, e.g., in refs. [4447 and its cosmological application was considered in refs. [48, 49], but actually $D$-term action is required to realize Starobinsky inflation [42, 50-53]. In the new-minimal SUGRA, embedding of Starobinsky model was studied in ref. [54] and reconsidered in the inflationary context with higher order corrections in Ricci scalar in ref. [55]. See also refs. [56, 57].

\footnotetext{
${ }^{2}$ In the case without any higher derivative terms, the equivalence between different formulations of SUGRA is shown from the conformal SUGRA viewpoint [36].
} 
Besides these pure SUGRA models without matter, there are many SUGRA models with matter that have Starobinsky-like scalar potentials (see, as an incomplete list, refs. [38, $39,42,50-52,55-61])$, so it is of prime importance to distinguish these models by studying cosmological scenarios after inflation. To discuss reheating of the universe, one has to couple the pure SUGRA inflation sector to matter sector. Ref. [62] studied soft SUSY breaking pattern in the old-minimal case, whereas ref. [63] discussed some features of matter-coupling in the new-minimal setup.

In this paper, we consider generic old-minimal SUGRA models [38, 40, 42] that realize Starobinsky inflation focusing particularly on the model in ref. [39]. One of the reasons for the choice of the old-minimal formulation is that one has eventually to break R-symmetry to give gauginos their masses, but the new-minimal formulation has an exact R-symmetry. We assume the absence of even higher order terms in scalar curvature, corresponding to absence of superderivatives in the SUGRA action, because such terms may modify or hamper inflation [64]. We introduce matter-coupling and study its cosmological consequences. In particular, we study various inflaton decay channels extensively. In contrast to the original non-SUSY version of reheating in the Starobinsky model, there is a long-lived particle, gravitino. The gravitino is the superpartner of the graviton and hence always present in SUGRA, and its abundance is a cosmologically important subject. We thus study the partial decay rate into gravitinos, and resultant constraints on parameters of the theory.

Before explaining our setup in section 2, we briefly emphasize the differences from the literature. In our setup, as we will see, the inflaton must have specific super- and Kähler potentials. For example, the exponential of Kähler potential linearly depends on the real part of the inflaton, while the gauge kinetic function never depends on the inflaton. Our setup, the old-minimal SUGRA realization of the Starobinsky model, is thus predictive. To the best of our knowledge, this is the first study of inflaton decay and gravitino production in the theory described by a modified action of supergravity. In section 3, we study various partial decay rates of the inflaton. In section 4, we discuss the cosmological constraints from gravitino abundance. We summarize and discuss differences from the original (nonSUSY) version of the Starobinsky model in section 5. The duality transformation between a higher derivative SUGRA and the corresponding standard SUGRA is reviewed, and some generalization of it is discussed in appendix A. We use the reduced Planck unit $c=\hbar=M_{\mathrm{G}}=1$ with $M_{\mathrm{G}}=M_{\mathrm{Pl}} / \sqrt{8 \pi}=1 / \sqrt{8 \pi G}$ unless otherwise stated, and basically use the notation and convention of ref. [65].

\section{Starobinsky model embedded in matter-coupled old-minimal super- gravity}

The Starobinsky model is based on a pure gravity action with a second order term of scalar curvature. In the supergravity side, a generic (super)gravitational action up to matter and (super)derivatives is

$$
S_{\text {grav }}=\int \mathrm{d}^{4} x \mathrm{~d}^{4} \theta E N(\mathcal{R}, \overline{\mathcal{R}})+\left[\int \mathrm{d}^{4} x \mathrm{~d}^{2} \Theta 2 \mathscr{E} F(\mathcal{R})+\text { H.c. }\right]
$$


where $\mathcal{R}$ is the curvature chiral superfield, $E$ is the full density, $\mathscr{E}$ is the chiral density, $\Theta$ is the so-called new $\Theta$ variable [65], $N(\mathcal{R}, \overline{\mathcal{R}})$ is a Hermitian function, and $F(\mathcal{R})$ is a holomorphic function. ${ }^{3}$

To discuss inflaton decay and reheating of the universe, we consider a simple way of coupling the above action to matter sector. We take the minimal coupling between the SUGRA sector described by the curvature chiral superfield $\mathcal{R}$ and the matter sector described by chiral superfields $\phi^{i}$ and vector superfields $V^{A}$ :

$$
\begin{aligned}
S= & \int \mathrm{d}^{4} x \mathrm{~d}^{4} \theta E\left(N(\mathcal{R}, \overline{\mathcal{R}})+J\left(\phi, \bar{\phi} e^{g V}\right)\right) \\
& +\left[\int \mathrm{d}^{4} x \mathrm{~d}^{2} \Theta 2 \mathscr{E}\left(F(\mathcal{R})+P(\phi)+\frac{1}{4} h_{A B}(\phi) W^{A} W^{B}\right)+\text { H.c. }\right] \\
= & \int \mathrm{d}^{4} x \mathrm{~d}^{4} \theta E N(\mathcal{R}, \overline{\mathcal{R}}) \\
& +\left[\int \mathrm{d}^{4} x \mathrm{~d}^{2} \Theta 2 \mathscr{E}\left(F(\mathcal{R})+\frac{3}{8}(\overline{\mathscr{D}} \overline{\mathscr{D}}-8 \mathcal{R}) e^{-K^{(\phi)} / 3}+P(\phi)+\frac{1}{4} h_{A B}(\phi) W^{A} W^{B}\right)+\text { H.c. }\right]
\end{aligned}
$$

where $g$ is the gauge coupling constant, $\phi$ collectively denotes $\phi^{i}$ 's, $J\left(\phi, \bar{\phi} e^{g V}\right)$ is a Hermitian function, $P(\phi)$ is a holomorphic function, and $K^{(\phi)}\left(\phi, \bar{\phi} e^{g V}\right)=-3 \ln \left(-\frac{J\left(\phi, \bar{\phi} e^{g V}\right)}{3}\right)$ is the Kähler potential of the matter fields.

The above action can be recast into the following form [38, 63]:

$$
S=\int d^{4} x d^{2} \Theta 2 \mathscr{E} \frac{3}{8}(\overline{\mathscr{D}} \overline{\mathscr{D}}-8 \mathcal{R}) e^{-K / 3}+W+\frac{1}{4} h_{A B} W^{A} W^{B}+\text { H.c. }
$$

with the Kähler potential and superpotential specified as follows,

$$
\begin{aligned}
& K=-3 \ln \left(\frac{T+\bar{T}-N(S, \bar{S})-J\left(\phi, \bar{\phi} e^{g V}\right)}{3}\right), \\
& W=2 T S+F(S)+P(\phi) .
\end{aligned}
$$

The derivation (in a more general setup) is reviewed in appendix A. Note that the dependence of these potentials on the inflaton $T$ is completely determined by the structure of the theory: the origin of the inflaton $T$ is the Lagrange multiplier. ${ }^{4}$ This structure is not altered even if non-minimal couplings between $\mathcal{R}$ and matter superfields, which we do not discuss in this paper, are introduced because they become non-minimal couplings between $S$ (but not $T$ ) and matter superfields in the transformed theory. ${ }^{5}$ Therefore, in

\footnotetext{
${ }^{3}$ The first, non-holomorphic term is called $D$-term action as it is from $D$-component of Kähler potential of $\mathcal{R}$, while the second, holomorphic term is called $F$-term action as it is from $F$-component of superpotential of $\mathcal{R}$.

${ }^{4}$ Recently, the work [66] suggested a higher derivative SUGRA model in which a superpotential term of $S$ and $T$ is given by $W=g(T) S$. Such a superpotential can be realized if $T$ is not a Lagrange multiplier but a chiral multiplet coupled to $\mathcal{R}$ and $\overline{\mathcal{R}}$ (see ref. [67] for an earlier discussion). We briefly discuss similar extensions in appendix A. In this work, we discuss the minimal case that the chiral multiplets $T$ and $S$ are purely originated from the gravitational multiplet and its higher derivative modes, and that the superpotential term of $T$ and $S$ is given by $W=2 T S$ as in eq. (2.5).

${ }^{5}$ We briefly discuss a possibility of $T$ dependent gauge kinetic functions in appendix A.
} 
this sense, the couplings between $T$ and matters discussed in this paper are universal in old-minimal Starobinsky inflation.

The Kähler metric and its inverse are given by

$$
\begin{gathered}
g_{I \bar{J}}=\frac{3}{(T+\bar{T}-N-J)^{2}}\left(\begin{array}{ccc}
1 & -N_{\bar{S}} & -J_{\bar{j}} \\
-N_{S} & N_{S \bar{S}}(T+\bar{T}-N-J)+N_{S} N_{\bar{S}} & N_{S} J_{\bar{j}} \\
-J_{i} & N_{\bar{S}} J_{i} & J_{i \bar{j}}(T+\bar{T}-N-J)+J_{i} J_{\bar{j}}
\end{array}\right), \\
g^{\bar{I} J}=\frac{T+\bar{T}-N-J}{3}\left(\begin{array}{rrr}
(T+\bar{T}-N-J)+N_{S} N^{S}+J_{k} J^{k} & N^{S} & J^{j} \\
N^{\bar{S}} & N^{\bar{S} S} & 0 \\
J^{\bar{i}} & 0 & J^{\bar{j} j}
\end{array}\right),
\end{gathered}
$$

where $I, J, \cdots=T, S, i, j, \ldots$ (or $\phi^{i}, \phi^{j}, \ldots$ ) are field indices, $N^{\bar{S} S}=\left(N_{S \bar{S}}\right)^{-1}, J^{\overline{i j}}$ is the inverse matrix of $J_{i \bar{j}}$, and indices are uppered and lowered by these matrices, e.g. $N^{S}=$ $N^{\bar{S} S} N_{\bar{S}}$ and $J^{\bar{i}}=J^{i j} J_{j}$. The scalar potential is

$$
\begin{aligned}
V=\left(\frac{3}{A}\right)^{2}( & N^{\bar{S} S}\left|2 T+F_{S}\right|^{2}+|2 S|^{2}\left(A+N_{S} N^{S}+J_{i} J^{i}\right)+\bar{P}_{i} J^{\bar{i} j} P_{j} \\
& \left.+\left\{2 \bar{S}\left[\left(2 T+F_{S}\right) N^{S}+P_{i} J^{i}-3 W\right]+\text { h.c. }\right\}\right)+\frac{g^{2}}{2} D^{A} D_{A}
\end{aligned}
$$

where we have defined a compact notation $A \equiv T+\bar{T}-N-J{ }^{6}$ Indices of D-terms, $D^{A}\left(D_{A}\right)$, are lowered (lifted) by (the inverse of) the real part of the gauge kinetic matrix function $h_{A B}^{R}\left(h_{R}^{A B}\right)$.

The inflaton (or SUGRA) sector ( $T$ and $S$ ) of this class of modified SUGRA models was studied in ref. [42]. The Starobinsky model is realized in this setup essentially as the modified Cecotti model [39]:

$$
\begin{aligned}
N(S, \bar{S}) & =-3+\frac{12}{m_{\Phi}^{2}} S \bar{S}-\frac{\zeta}{m_{\Phi}^{4}}(S \bar{S})^{2}, \\
F(S) & =0
\end{aligned}
$$

where $m_{\Phi}$ is the inflaton mass at the vacuum, and $\zeta(>0)$ gives a SUSY-breaking mass to $S$ and stabilizes its potential. The real part of $T$ becomes the inflaton, and the canonically normalized scalar potential is that of the Starobinsky model, $V=\frac{3 m_{\Phi}^{2}}{4}\left(1-e^{-\sqrt{2 / 3} \widehat{\operatorname{Re} T}}\right)^{2}$, where $\widehat{\operatorname{Re} T} \equiv-K / \sqrt{6}$ is the canonically normalized inflaton field (during inflation). $S$ is the sGoldstino field that breaks SUSY during inflation. At the vacuum $(T=S=0)$, SUSY is preserved.

Introduction of the linear term in $S / m_{\Phi}$ into eq. (2.9) can make SUSY breaking vacua with an almost vanishing cosmological constant without spoiling inflation [70]. This is an interesting possibility because the higher derivative version of the purely supergravitational theory describes not only the inflation but also SUSY breaking. However, the SUSY

\footnotetext{
${ }^{6}$ It is often denoted as $\Omega=-3 A$ in the standard notation [65], and $\tilde{\phi}=-3 A$ in the conformal SUGRA notation [68]. The functional form of $\Omega$ is important for the SUSY breaking effects on inflationary dynamics [69].
} 
breaking scale becomes the inflation scale $\left(m_{\Phi} \sim 10^{13} \mathrm{GeV}\right)$, which typically makes the Higgs particle too heavy [71]. Although the tree-level contributions to soft SUSY breaking parameters can be suppressed by assuming a minimal coupling between the MSSM sector and the SUGRA sector as in our setup, there are anomaly-mediated contributions to gaugino masses, which in turn give other particles their masses through renormalization group running.

Therefore, we concentrate on models that deviate (if any) only slightly from the simple model (2.9), (2.10). For definiteness, we assume $\left|N^{S}\right|$ and $\left|N^{\bar{S} S} F_{S S}\right|$ are at most of order the gravitino mass $m_{3 / 2}$, which is supposed to be much smaller than the inflaton mass, $m_{3 / 2} \ll m_{\Phi}$. Perturbation by higher order terms are negligible because VEV of $S$ is suppressed. ${ }^{7}$ Since the inflaton sector does not break SUSY at the vacuum, we introduce a hidden SUSY breaking sector. We treat the SUSY breaking sector as general as possible, but occasionally we assume a simple SUSY breaking sector described by

$$
\begin{gathered}
J(z, \bar{z})=|z|^{2}-\frac{|z|^{4}}{\Lambda^{2}}, \\
P(z)=\mu^{2} z+W_{0},
\end{gathered}
$$

where $J(z, \bar{z})$ and $P(z)$ are the Kähler potential and superpotential of the SUSY breaking field $z$ [see equations (2.4) and (2.5)]. We also assume that VEVs of $\phi^{i}, J\left(\phi^{i}, \bar{\phi}^{\bar{j}}\right), P\left(\phi^{i}\right)$, and their derivatives are negligibly small except for those of SUSY breaking field $z$, which is easily satisfied if $\phi^{i}$ 's are charged under some unbroken symmetry.

All of the four scalar degrees of freedom and four fermionic degrees of freedom in the inflaton sector are degenerate in their masses $\left(=m_{\Phi}\right)$ at the zeroth order of perturbation with respect to SUSY breaking $\left(m_{3 / 2}\right)$. In the scalar sector, imaginary parts of $T$ and $S$ are still degenerate at the first order of gravitino mass, but the sum and difference of real parts of $T$ and $S$ have mass eigenvalues $m_{\Phi} \mp m_{3 / 2}$. Also, $S$ acquires its VEV, $\langle S\rangle=\langle W\rangle / 2$. Here we have neglected supersymmetric mass term of $S$ from its superpotential, $F_{S S}$. Fermionic mass eigenvalues depend on the detail of functions $N$ and $F$, but in the simplest case (2.9), (2.10), they are still degenerate at the first order in gravitino mass. For this kinematical reason, the decay of inflaton into particles in the inflaton sector (inflatino and gravitino), if possible, is extremely suppressed.

The mass eigenstates of the canonically normalized scalar linear fluctuations are approximately given by

$$
\Phi_{R \pm}=\frac{\sqrt{g_{T \bar{T}}}}{2}(T+\bar{T}) \pm \frac{\sqrt{g_{S \bar{S}}}}{2}(S+\bar{S}) \simeq \frac{1}{2 \sqrt{3}}(T+\bar{T}) \pm \frac{\sqrt{3}}{m_{\Phi}}(S+\bar{S}) .
$$

Because the $T$-S oscillation time scale $\tau_{\text {osc }} \sim\left(2 m_{3 / 2}\right)^{-1}$ is much shorter than the lifetime $\tau_{\mathrm{dec}} \sim\left(M_{\mathrm{G}}^{2} / m_{\Phi}^{3}\right)$ for gravitino mass above $\mathrm{GeV}$ scale, decay rates from these mass eigenstates are appropriate quantities. However, the interactions are simply described in the basis of $T$ and $S$ but not of their linear combination, so for simplicity of presentation we

\footnotetext{
${ }^{7}$ Although it vanishes at the leading order, it has a value of the order of the gravitino mass after SUSY breaking. See the following discussion.
} 
describe partial decay rates of inflaton in the next section as if $T$ (or $S$ ) is the parent particle. The true rates are the averages of those for $T$ and $S$.

We take gravitino mass larger than $\mathrm{TeV}$ scale because we assume anomaly (or gravity) mediation of SUSY breaking, in which SUSY breaking is transmitted to the visible sector by the Planck suppressed coupling to the auxiliary field of the curvature superfield $\mathcal{R}$ in the transformed theory (2.3) due to the trace anomaly (or by the Planck suppressed coupling to the hidden sector in the tree-level potential). We respect the philosophy of the Starobinsky model in this paper, that is, we exploit the (super-)gravitational sector as much as possible, and do not introduce an inflaton nor messenger fields by hand.

\section{Inflaton decay}

Various modulus/inflaton decay modes and their cosmological consequences have been extensively studied in ref. [72]. Inflaton decay in the case of no-scale supergravity has also been studied in ref. [73], but in our case inflaton has supergravitational origin so that the form of inflaton Kähler potential is different from that in ref. [73]. Moreover, these works suppose that the inflaton mass $m_{\Phi}$ comes mainly from the second derivative $W_{\Phi \Phi}$ of the superpotential with respect to the inflaton $\Phi$ itself. In our case, on the other hand, the origin of the inflaton mass $m_{T}\left(\equiv m_{\Phi}\right)$ is from $W_{T S}$ rather than $W_{T T}$. We study inflaton decay in our setup taking these differences into account.

At the end of inflation, the inflaton oscillates around the minimum of the potential for a long time due to its Planck-suppressed decay rate. We have numerically checked that the energy stored in $\operatorname{Re} T$ does not flow into $\operatorname{Im} T$ or $S$ fields in this classical oscillation dynamics. In the following, we study various partial decay rates of the inflaton at the treelevel unless the one-loop process becomes leading. As stated at the end of the previous section, we first consider interactions involving $T$, followed by similar analyses for $S$.

\subsection{Two-body decay of $T$ into scalars, spinors and gauge bosons}

\subsubsection{Decay into scalars}

It is convenient to define the reduced scalar potential $\tilde{V}$ as $V=\left(\frac{3}{A}\right)^{2} \tilde{V}+\frac{g^{2}}{2} D^{A} D_{A}$, or equivalently,

$$
\begin{aligned}
\tilde{V}= & N^{\bar{S} S}\left|2 T+F_{S}\right|^{2}+|2 S|^{2}\left(A+N_{S} N^{S}+J_{i} J^{i}\right)+\bar{P}_{i} J^{\bar{i} j} P_{j} \\
& +\left\{2 \bar{S}\left[\left(2 T+F_{S}\right) N^{S}+P_{i} J^{i}-3 W\right]+\text { h.c. }\right\} .
\end{aligned}
$$

Although $T$ and $S$ are singlets, derivatives of the $D$-term with respect to them are nonzero,

$$
\begin{aligned}
& D_{A T}=-i g_{T \bar{i}} \bar{X}_{A}^{\bar{i}}=\frac{3}{A^{2}} i J_{\bar{i}} \bar{X}_{A}^{\bar{i}}=-\frac{1}{A} G_{\bar{i}} D_{A} \bar{i}=-\frac{1}{A} D_{A} \simeq-\frac{1}{3} D_{A}, \\
& D_{A S}=-i g_{S \bar{i}} \bar{X}_{A}^{\bar{i}}=-\frac{3}{A^{2}} i N_{S} J_{\bar{i}} \bar{X}_{A}^{\bar{i}}=\frac{1}{A} N_{S} G_{\bar{i}} D_{A} \overline{\bar{i}}=\frac{1}{A} N_{A} D_{A} \simeq \frac{1}{3} N_{S} D_{A},
\end{aligned}
$$

where $G=K+\ln |W|^{2}$ is the total Kähler potential, $X_{A}$ is the Killing vector of the Kähler manifold, and we have used the gauge symmetry of the superpotential. With the aid of 
the condition of the vanishing cosmological constant, $V=0$, the stationary conditions for $T$ and $S$ at the vacuum, $V_{T}=V_{S}=0$, reduce to $\tilde{V}_{T}=\tilde{V}_{S}=0$.

Using the above formulas and the facts $\tilde{V}_{T T}=\tilde{V}_{T i}=\tilde{V}_{T \bar{i}}=0$, the relevant vertex functions are derived as

$$
V_{\tilde{T} i \tilde{j}}=-\frac{2}{A} V_{\tilde{i} \tilde{j}} \simeq-\frac{2}{3} V_{\tilde{i} \tilde{j}}
$$

where tilded indexes may take both of holomorphic and anti-holomorphic indexes like $\tilde{I}=I, \bar{I}$. This means that the interaction terms are proportional to the mass terms of scalars. There is a same order contribution from the kinetic term. Combining mass and kinetic term contributions, the rate is

$$
\Gamma\left(T \rightarrow \phi^{i} \bar{\phi}^{\bar{i}}\right)=\frac{3 m_{i}^{4}}{8 \pi M_{\mathrm{G}}^{2} m_{\Phi}},
$$

where $m_{i}$ is the mass of the daughter particle $\phi^{i}$. The kinetic term also provides the $\phi^{i} \phi^{j}$ production process with the rate

$$
\Gamma\left(T \rightarrow \phi^{i} \phi^{j}\right)=\frac{m_{\Phi}^{3}}{96 \pi M_{\mathrm{G}}^{2}}\left|J_{i j}\right|^{2} .
$$

The partial decay rates of inflaton into $\operatorname{Im} T, S$, or $\bar{S}$ and $\phi^{i}$ are suppressed by $J_{i}$ and phase space factors.

\subsubsection{Decay into spinors}

It is convenient to define the reduced fermion mass matrix $\tilde{M}$ as $M_{I J}=e^{G / 2} \tilde{M}_{I J}$, where $M_{I J}$ is the fermion mass matrix, or equivalently,

$$
\tilde{M}_{I J}=\nabla_{I} G_{J}+G_{I} G_{J}-\frac{2}{3}\left(\left\langle G_{I}\right\rangle G_{J}+\left\langle G_{J}\right\rangle G_{I}\right)+\frac{2}{3}\left\langle G_{I} G_{J}\right\rangle .
$$

Terms with VEVs are induced by the redefinition of the gravitino field to absorb goldstino into gravitino. The inflaton-spinor-spinor vertex is obtained by differentiating the mass matrix, $M_{I J T}=G_{T} M_{I J} / 2+e^{G / 2} \tilde{M}_{I J T} \simeq m_{3 / 2} \tilde{M}_{I J T}$. Under the approximation like $A \simeq 3$ and $S \simeq W / 2$, and neglecting $G_{i}, G_{T}$ and $G_{S}$, the reduced fermion matrix $\tilde{M}_{i j}$ is approximated as $\tilde{M}_{i j} \simeq P_{i j} / W+J_{i j}-J_{i j \bar{z}} G^{\bar{z}}$ where $z$ is the SUSY breaking field. Under the same approximation,

$$
\tilde{M}_{i j T} \simeq-\tilde{M}_{i j}
$$

On the other hand, $\tilde{M}_{i j \bar{T}}$ vanishes at the vacuum. The kinetic term gives a same order contribution. Combining the mass and kinetic term contributions, the partial decay rate is expressed as

$$
\Gamma\left(T \rightarrow \chi^{i} \bar{\chi}^{\bar{i}}\right)=\frac{m_{i}^{2} m_{\Phi}}{192 \pi M_{\mathrm{G}}^{2}},
$$

where $m_{i}$ is the mass of the spinor $\chi^{i}$. We have assumed here that the mixing terms between matter spinors and gauginos are smaller than the diagonal parts, $\left|M_{I A}\right| \ll\left|M_{J K}\right|$.

The partial decay rates of inflaton into inflatino or $S$-ino and $\chi^{i}$ are suppressed by $J_{i}$ and phase space factor. 


\subsubsection{Anomaly-induced decay into gauge sector}

The inflaton $T$ has the Lagrange multiplier origin so that it never appears in the gauge kinetic function. We have to consider decay into gauge sector via the anomaly-induced one loop process $[72,74]$ unless we introduce a non-minimal term depending on $W^{A}$ in the $D$-term action (see appendix A). The rate is $[72,74]$

$$
\Gamma(T \rightarrow A A)+\Gamma(T \rightarrow \lambda \lambda) \simeq \frac{N_{\mathrm{g}} \alpha^{2}}{256 \pi^{3}}\left|X_{G}\right|^{2} m_{\Phi}^{3}
$$

where $N_{\mathrm{g}}$ and $\alpha$ are the number of the generators and the fine structure constant of the gauge group, $X_{G}=\sqrt{6}\left[\left(T_{G}-T_{R}\right) K_{T}+\frac{2 T_{R}}{d_{R}}\left(\left.\log \operatorname{det} K\right|_{R} ^{\prime \prime}\right)_{T}\right], T_{G}$ and $T_{R}$ are the Dynkin indexes of the adjoint representation and representation $R, d_{R}$ is the dimension of the representation $R$, and $\left.K\right|_{R} ^{\prime \prime}$ is the Kähler metric restricted to the matter whose representation is $R$. In our case, the rate becomes (also see $[75,76]$ for non-SUSY case)

$$
\Gamma(T \rightarrow A A)+\Gamma(T \rightarrow \lambda \lambda) \simeq \frac{3 N_{\mathrm{g}} \alpha^{2} m_{\Phi}^{3}}{128 \pi^{3} M_{\mathrm{G}}^{2}}\left(T_{G}-\frac{1}{3} T_{R}\right)^{2} .
$$

\subsection{Three-body decay of $T$}

Let us first consider the decay channel into a scalar and two spinors involving Yukawa coupling. There are three diagrams at the tree level that are of the same order. The effective interaction term that reproduces the decay rate is found to be [72]

$$
\mathcal{L}_{\text {eff }} \simeq-\frac{1}{2} e^{G / 2}\left(G_{T i j k}-3 \Gamma_{T(i}^{l} G_{j k) l}\right) T \phi^{i} \chi^{j} \chi^{k}+\text { h.c. }
$$

In our case, the leading terms, which could lead to the typical Planck-suppressed decay rate, cancel each other, and the remaining terms give at most $\Gamma \sim m_{3 / 2}^{2} m_{\Phi}^{3} / M_{\mathrm{G}}^{4}$.

There are also scalar three-body decay. At the vacuum, the scalar four-point vertex is given by

$$
V_{\tilde{T} \tilde{i} \tilde{j} \tilde{k}} \simeq-\frac{2}{3}\left(\tilde{V}_{\tilde{i} \tilde{j} \tilde{k}}+V_{D \tilde{i} \tilde{j} \tilde{k}}\right)
$$

The leading terms in $\tilde{V}_{i j k}$ cancel each other in the same way as for the above fermion case.

$$
\begin{aligned}
& \tilde{V}_{i j k} \simeq-3 \bar{P}^{z} J_{z \bar{m}(i} J^{\bar{m} l} P_{j k) l}, \\
& \tilde{V}_{i j \bar{k}} \simeq \bar{P}_{\overline{l k}} J^{\bar{l} l} P_{i j l}-\bar{P}^{m} J_{m \bar{n} \bar{k}} J^{\bar{n} l} P_{i j l}+2 \bar{S} P_{i j l} J^{\overline{l l}}\left(J_{\overline{l k}}-J_{\bar{l} m \bar{k}} J^{m}\right) .
\end{aligned}
$$

The rates are suppressed by gravitino or matter mass squared, $\Gamma \sim m_{X}^{2} m_{\Phi} / M_{\mathrm{G}}^{2}$ with $m_{X}=\max \left[m_{(\text {matter })}, m_{3 / 2}\right]$ at most.

We also considered decay modes involving $\operatorname{Im} T, S$, or their superpartners, but these rates are at most of order of $m_{\Phi}^{5} / M_{\mathrm{G}}^{4}$ with additional phase space suppression. Four- or more- body decay rates are more suppressed by the phase space factor. 


\subsection{Decay of $S$}

In the same way as the previous subsections, we study decay channel of $S$ in this subsection. Although $S$ is basically conformally sequestered from the matter sector in our setup, it has unsupressed coupling with $T$ in the superpotential, which in turn couples to the matter sector universally. Consequently, $S$ has unsuppressed coupling to matter in some decay channels. Important partial decay rates are as follows,

$$
\begin{aligned}
\Gamma\left(S \rightarrow \phi^{i} \phi^{i}\right) & \simeq \frac{m_{i}^{2} m_{\Phi}}{48 \pi M_{\mathrm{G}}^{2}} \\
\Gamma\left(\bar{S} \rightarrow \chi^{i} \chi^{j}\right) & \simeq \frac{m_{\Phi}^{3}}{48 \pi M_{\mathrm{G}}^{2}}\left|J_{i j}\right|^{2}
\end{aligned}
$$

Beware $m_{S}=m_{\Phi}$. The above rates are calculated expanding mass terms. If there are no heavy matter particles, the following contribution from kinetic term becomes important,

$$
\Gamma\left(S \rightarrow \phi^{i} \phi^{j}\right) \simeq \frac{m_{\Phi} m_{3 / 2}^{2}}{192 \pi M_{\mathrm{G}}^{2}}\left|J_{i j}\right|^{2},
$$

while other channels $\Gamma\left(S \rightarrow \phi^{i} \bar{\phi}^{\bar{j}}\right)$ and $\Gamma\left(S \rightarrow \chi^{i} \chi^{j}\right)$ from kinetic terms are suppressed by both of $N_{S}$ and matter masses. For decay modes of $S$ involving $T$, see the previous subsections, $\Gamma(S \rightarrow T X)=\Gamma(T \rightarrow S X)$. The anomaly-induced decay of $S$ involves an additional $-N_{S}$ factor compared to the case of $T$.

\subsection{Gravitino production}

In this subsection we study gravitino production from the inflaton decay, which is one of the distinguishing features from the non-SUSY version of the Starobinsky model. Although we have treated $T$ or $S$ as the parent particle in the previous subsections, the mixing effect is essential in gravitino production [77, 78]. We thus use the proper mass eigenstates $(2.13)$ in evaluating the inflaton decay rate into gravitinos.

\subsubsection{Single gravitino production}

The partial decay rate of a scalar particle into its superpartner and a gravitino is calculated, e.g., in ref. [79]. Because inflaton and inflatino are degenerate before SUSY breaking, their mass splitting is of the order of gravitino mass. We parametrize the mass difference as $m_{\Phi}-m_{\tilde{\Phi}}=\Delta m_{3 / 2}$. The decay rate is approximately

$$
\Gamma\left(\Phi \rightarrow \tilde{\Phi} \psi_{3 / 2}\right) \simeq \frac{m_{\Phi}^{3}}{3 \pi M_{\mathrm{G}}^{2}}\left(\frac{m_{3 / 2}}{m_{\Phi}}\right)^{2} \Delta\left(\Delta^{2}-1\right)^{\frac{3}{2}},
$$

where we explicitly wrote the reduced Planck mass $M_{\mathrm{G}}$. Thus, the single gravitino production has the suppression factor $\left(m_{3 / 2} / m_{\Phi}\right)^{2}$ and $\left(\Delta^{2}-1\right)^{3 / 2}$ compared to the typical Planck-suppressed decay rate $\mathcal{O}\left(m_{\Phi}^{3} / M_{\mathrm{G}}^{2}\right)$. In subsequent discussion, we neglect the single gravitino production rate because it is at most of order of gravitino pair production rate discussed below. 


\subsubsection{Gravitino pair production}

Gravitino pair production rate from modulus/inflaton decay has been extensively studied in the literature $[77,78,80-82]$. See also refs. [72, 74, 83-86] for other decay channels and cosmological consequences.

The gravitino pair production rate from a mass eigenstate $\Phi$ is given by $[77,78,80-82]$

$$
\Gamma\left(\Phi \rightarrow \psi_{3 / 2} \psi_{3 / 2}\right)=\frac{\left|\mathcal{G}_{\Phi}^{(\text {eff })}\right|^{2} m_{\Phi}^{5}}{288 \pi m_{3 / 2}^{2}},
$$

where the mass hierarchy $m_{\Phi} \gg m_{3 / 2}$ is assumed, and the effective coupling is given by [82] $\left|\mathcal{G}_{\Phi}^{\text {(eff) }}\right|^{2}=2\left|G_{I}\left(\mathcal{A}^{-1}\right)^{I} \Phi\right|^{2}$, where $\mathcal{A}$ is the mixing matrix [82]. In our case, the inflaton is the real part of $T$, but the real parts of $T$ and $S$ mix almost maximally at the vacuum (see eq. (2.13)).

Because the SUSY breaking of $T$ and $S$ are small, $\left|G_{T}\right|,\left|G_{S}\right| \ll 1$, the effective coupling reduces to

$$
\left|\mathcal{G}_{\Phi_{\mathrm{R} \pm}}^{(\mathrm{eff})}\right|^{2}=2\left|\frac{\sqrt{3}}{2} G_{T} \pm \frac{m_{\Phi}}{4 \sqrt{3}} G_{S}+\left(\mathcal{A}^{-1}\right)^{i} \Phi_{\mathrm{R} \pm} G_{i}\right|^{2} .
$$

We will first evaluate $G_{T}$ and $G_{S}$, and then proceed to $\left(\mathcal{A}^{-1}\right)^{i}{ }_{\Phi_{\mathrm{R}}}$. We evaluate $G_{T}$ using the conditions $V=e^{G}\left(G_{I} G^{I}-3\right)+\left(g^{2} / 2\right) D^{A} D_{A}=0$ for the vanishing cosmological constant and $V_{\bar{I}}=e^{G}\left(G_{\bar{I}} G^{J} G_{J}-2 G_{\bar{I}}+G^{\bar{J}} \nabla_{\bar{I}} G_{\bar{J}}\right)+g^{2}\left(-\left(h_{A B I}^{R} / 2\right) D^{A} D^{B}+D^{A} D_{A I}\right)=0$, where $\nabla_{I} G_{J}=G_{I J}-G_{I J \bar{K}} G^{\bar{K}}$, for the stationarity of the potential at the vacuum. The relevant equations are

$$
\begin{aligned}
G_{\bar{T}}+G^{\bar{I}} \nabla_{\bar{T}} G_{\bar{I}} & =3 \delta\left(G_{\bar{T}}+\frac{2}{3}\right), \\
G_{\bar{S}}+G^{\bar{I}} \nabla_{\bar{S}} G_{\bar{I}} & =3 \delta\left(G_{\bar{S}}-\frac{2}{3} N_{\bar{S}}\right),
\end{aligned}
$$

where $\delta=\frac{g^{2}}{6 m_{3 / 2}^{2}} D^{A} D_{A}$ is the D-term SUSY breaking fraction. More explicitly, eq. (3.22) is

$$
3 \delta G_{\bar{T}}+2 \delta=G_{\bar{T}}+G_{T} g^{\bar{I} T} \nabla_{\bar{T}} G_{\bar{I}}+G_{i} g^{\bar{J} i} \nabla_{\bar{T}} G_{\bar{J}}+G_{S} g^{\bar{I} S} \nabla_{\bar{T}} G_{\bar{I}} .
$$

We concentrate on models that deviate only slightly from the simple model (2.9), (2.10), so we assume $\left|N_{S}\right|$ and $\left|F_{S S}\right|$ are at most of order $m_{3 / 2} / m_{\Phi}^{2}$. We also use $S \simeq W / 2$. For example,

$$
G_{T i \bar{j}}=-\frac{3 J_{i \bar{j}}}{(T+\bar{T}-N-J)^{2}}-\frac{6 J_{i} J_{\bar{j}}}{(T+\bar{T}-N-J)^{3}} \simeq-\frac{1}{3} J_{i \bar{j}} .
$$

Similarly, $\nabla_{T} G_{T} \simeq-2 / 3, \nabla_{T} G_{S} \simeq 2 / W$, and $\nabla_{T} G_{i} \simeq-2 G_{i} / 3$. Equation (3.22) becomes

$$
0 \simeq(1-3 \delta) G_{\bar{T}}+2\left(\frac{N^{\bar{S}}}{\bar{W}}-1\right) G_{T}-2+2 G_{S} \frac{N^{\bar{S} S}}{\bar{W}} \simeq-2+2 G_{S} \frac{N^{\bar{S} S}}{\bar{W}},
$$

so $G_{S}$ is approximately given by

$$
G_{S} \simeq N_{S \bar{S}} \bar{W} \simeq \frac{12 m_{3 / 2}}{m_{\Phi}^{2}}
$$


This implies the tiny VEV of $T$ :

$$
T \simeq \frac{3 m_{3 / 2}^{2}}{m_{\Phi}^{2}}-\frac{F_{S}}{2} .
$$

In the same way, from eq. (3.23), $\nabla_{S} G_{S} \simeq F_{S S} / W$ and $\nabla_{S} G_{i} \simeq-N_{S} G_{i} / 3$, we obtain

$$
0 \simeq(1-3 \delta) G_{\bar{S}}+\left(\frac{2 N^{S}+N^{\bar{S} S} \bar{F}_{\bar{S} \bar{S}}}{\bar{W}}\right) G_{S}+\left(\frac{6+N^{\bar{S}} \bar{F}_{\bar{S} \bar{S}}}{\bar{W}}\right) G_{T}+\frac{2 J^{i}}{\bar{W}} G_{i}+2 N_{\bar{S}} .
$$

To simplify the expression, let us assume $F_{S S}=\delta=0, N=-3+\frac{12}{m_{\Phi}^{2}} S \bar{S}$ with $S=W / 2$ at the vacuum. Then, $G_{T}$ becomes

$$
G_{T} \simeq-6 \frac{m_{3 / 2}^{2}}{m_{\Phi}^{2}}-\frac{1}{3} J^{i} G_{i} .
$$

If we further assume for the SUSY breaking sector that $J(z, \bar{z})=|z|^{2}-\frac{|z|^{4}}{\Lambda^{2}}$ and $P(z)=\mu^{2} z+$ $W_{0}, J^{i}$ is given by $J^{z} \simeq|z|\left(1+\frac{2}{\Lambda^{2}}|z|^{2}\right) \simeq|z| \simeq \sqrt{12}\left(\frac{m_{3 / 2}}{m_{z}}\right)^{2}$, where $m_{z} \simeq \sqrt{12} m_{3 / 2} / \Lambda$. It is implied that

$$
S \simeq \frac{W}{2}\left(\frac{3}{A}+G_{T}\right)=\frac{W}{2}+\mathcal{O}\left(\frac{m_{3 / 2}^{3}}{m_{\Phi}^{3}} \text { or } \frac{m_{3 / 2}^{3}}{m_{\Phi} m_{z}^{2}}\right) .
$$

Equations (3.28) and (3.31) can be used to obtain shifts of quantities e.g. $A \simeq 3+3 m_{3 / 2}^{2} / m_{\Phi}^{2}$ induced by SUSY breaking.

The mixing matrix $\mathcal{A}$ has two effects: canonicalization of kinetic terms and diagonalization of mass terms. We assume that there is a single SUSY breaking field $\phi^{z}=z$, and its kinetic term and mass term are dominated by the diagonal part (proportional not $z z$ nor $\bar{z} \bar{z}$ but to $z \bar{z}$ ) for simplicity, and then the matrix element is simplified [82]

$$
\left(\mathcal{A}^{-1}\right)^{z}{ }_{\Phi_{\mathrm{R}} \pm}=\frac{g^{\bar{z} z}}{m_{\Phi}^{2}-m_{z}^{2}}\left(\frac{\sqrt{3}}{2}\left(V_{T \bar{z}}+V_{\bar{T} \bar{z}}+J_{\bar{z}}\left(V_{T \bar{T}}+V_{\bar{T} \bar{T}}\right)\right) \pm \frac{m_{\Phi}}{4 \sqrt{3}}\left(V_{S \bar{z}}+V_{\bar{S} \bar{z}}+J_{\bar{z}}\left(V_{S \bar{T}}+V_{\bar{S} \bar{T}}\right)\right)\right) .
$$

For the former part regarding $T$, only the $V_{T \bar{T}} \simeq 4 N^{\bar{S} S}$ term remains. If $m_{\Phi}^{2} \gg m_{z}^{2}$, this term cancels the term in $G_{T}$ proportional to $G_{z}$. For the latter part regarding $S$, all the four terms are nonzero:

$$
\begin{aligned}
\tilde{V}_{T S} & =-8 N^{\bar{S} S} N_{S S \bar{S}}|S|^{2}-4 \bar{S} \\
\tilde{V}_{T \bar{S}} & =-8 N^{\bar{S} S} N_{S \bar{S} \bar{S}}|S|^{2}+2 N^{\bar{S} S}\left(\bar{F}_{\bar{S} \bar{S}}+2 N_{\bar{S}}+2 \bar{S} N_{\bar{S} \bar{S}}\right)-8 S \\
\tilde{V}_{S \bar{z}}+\tilde{V}_{\bar{S} \bar{z}} & =\left(4(S+\bar{S}) J^{\bar{k}}+2 P_{l} J^{l \bar{k}}\right)\left(J_{\bar{k} \bar{z}}-J_{\bar{z} k \bar{k}} J^{k}\right)-4 \bar{P}_{\bar{z}}+2 \bar{P}_{\bar{k} \bar{z}} J^{\bar{k}}-2 \bar{P}_{\bar{k}} J^{\bar{k} l} J_{l \bar{m} \bar{z}} J^{\bar{m}},
\end{aligned}
$$

at the vacuum. Among these, $-4 \bar{P}_{\bar{z}}$ cancels the leading term in $G_{S}=12 m_{3 / 2} / m_{\Phi}+\cdots$ under the same condition $m_{\Phi}^{2} \gg m_{z}^{2}$. Assuming $J(z, \bar{z})=|z|^{2}-\frac{|z|^{4}}{\Lambda^{2}}$ and $P(z)=\mu^{2} z+W_{0}$, subleading terms regarding this cancellation are still subdominant compared to terms in $G_{T}$.

In summary, the effective coupling is approximated as

$$
\left|\mathcal{G}_{\Phi_{\mathrm{R} \pm}}^{(\mathrm{eff})}\right|^{2} \simeq 2\left|\frac{\sqrt{3}}{2}\left(-6 \frac{m_{3 / 2}^{2}}{m_{\Phi}^{2}}+\frac{1}{3} J^{z} G_{z} \frac{m_{z}^{2}}{m_{\Phi}^{2}-m_{z}^{2}}\right) \pm \frac{m_{\Phi}}{4 \sqrt{3}}\left(\frac{12 \bar{W}}{m_{\Phi}^{2}}-\frac{4 G^{z} G_{z} \bar{W}}{m_{\Phi}^{2}-m_{z}^{2}}\right)\right|^{2}
$$




$$
\simeq 6\left|3 \frac{m_{3 / 2}^{2}}{m_{\Phi}^{2}}+\frac{m_{z}^{2}}{m_{z}^{2}-m_{\Phi}^{2}}\left(\frac{1}{6} J^{z} G_{z} \mp \frac{\bar{W}}{m_{\Phi}}\right)\right|^{2} .
$$

Finally, the effective coupling is simplified when $m_{z}$ is in particular ranges:

$$
\left|\mathcal{G}_{\Phi_{\mathrm{R} \pm}}^{\text {(eff })}\right|^{2} \simeq \begin{cases}96\left(\frac{m_{3 / 2}}{m_{\Phi}}\right)^{4} & \left(m_{z}^{2} \ll m_{\Phi} m_{3 / 2}\right) \\ 6\left(\frac{m_{z}^{2} m_{3 / 2}}{m_{\Phi}^{3}}\right)^{2} & \left(3 m_{\Phi} m_{3 / 2} \ll m_{z}^{2} \ll m_{\Phi}^{2}\right) \\ 6\left(\frac{m_{3 / 2}}{m_{\Phi}}\right)^{2} & \left(m_{\Phi}^{2} \ll m_{z}^{2}\right)\end{cases}
$$

where we have assumed again $J(z, \bar{z})=|z|^{2}-\frac{|z|^{4}}{\Lambda^{2}}$ and $P(z)=\mu^{2} z+W_{0}$ to evaluate $J^{z} G_{z}$. Therefore, the gravitino pair production rate is

$$
\Gamma\left(\Phi_{\mathrm{R} \pm} \rightarrow \psi_{3 / 2} \psi_{3 / 2}\right) \simeq \frac{m_{\Phi}^{3}}{48 \pi M_{\mathrm{G}}^{2}} \times \begin{cases}16\left(\frac{m_{3 / 2}}{m_{\Phi}}\right)^{2} & \left(m_{z}^{2} \ll m_{\Phi} m_{3 / 2}\right) \\ \left(\frac{m_{z}}{m_{\Phi}}\right)^{4} & \left(3 m_{\Phi} m_{3 / 2} \ll m_{z}^{2} \ll m_{\Phi}^{2}\right) . \\ 1 & \left(m_{\Phi}^{2} \ll m_{z}^{2}\right)\end{cases}
$$

\section{Constraints from gravitino abundance}

We study gravitino abundance produced during and after reheating of the universe. Gravitino is generated by various processes, (i) direct decay of the inflaton, (ii) scattering in the thermal bath created by the inflaton decay, (iii) decay of particles such as $\chi^{S}$ and $z$ produced by inflaton decay, and (iv) decay of coherent oscillation of SUSY breaking field $z$. Similar analyses have been done in the literature, see refs. [87-89] and references therein.

As for direct decay of inflaton (i), we have derived various partial decay rates in the previous sections. We assume no significant entropy dilution occurs after the reheating of the universe due to the inflaton decay. Note that the SUSY breaking field $z$ decays dominantly into a pair of gravitinos, so that it does not produce entropy when it decays. We parametrize the total decay rate of inflaton as

$$
\Gamma_{\text {tot }}=X \frac{m_{\Phi}^{3}}{M_{\mathrm{G}}^{2}},
$$

where $X$ is defined by this equation. Among various decay channels, there is a generic decay channel via the anomaly-induced process. If we assume that this is the dominant mode, then $X$ is expressed as $X=N_{\mathrm{g}} \alpha^{2} b_{0}^{2} / 768 \pi^{3}$ where $b_{0}=3 T_{G}-T_{R}$. The branching ratio of the gravitino pair production is

$$
\operatorname{Br}\left(\Phi_{\mathrm{R} \pm} \rightarrow \psi_{3 / 2} \psi_{3 / 2}\right) \simeq \frac{1}{48 \pi X} \times \begin{cases}16\left(\frac{m_{3 / 2}}{m_{\Phi}}\right)^{2} & \left(m_{z}^{2} \ll m_{\Phi} m_{3 / 2}\right) \\ \left(\frac{m_{z}}{m_{\Phi}}\right)^{4} & \left(3 m_{\Phi} m_{3 / 2} \ll m_{z}^{2} \ll m_{\Phi}^{2}\right) \\ 1 & \left(m_{\Phi}^{2} \ll m_{z}^{2}\right)\end{cases}
$$

The gravitino yield $Y_{3 / 2} \equiv \frac{n_{3 / 2}}{s}$ where $n_{3 / 2}$ is gravitino number density and $s$ is entropy density, due to direct decay of inflaton is given by

$$
Y_{3 / 2}^{(\text {direct })}=\frac{3 T_{\mathrm{R}} \mathrm{Br}_{3 / 2}}{2 m_{\Phi}}
$$


where $\mathrm{Br}_{3 / 2}$ is the branching ratio into a gravitino pair, and we define the reheating temperature $T_{\mathrm{R}}$ as

$$
T_{\mathrm{R}}=\left(\frac{90}{\pi^{2} g_{*}\left(T_{\mathrm{R}}\right)}\right)^{\frac{1}{4}} \sqrt{M_{\mathrm{G}} \Gamma_{\mathrm{tot}}}
$$

The gravitino yield becomes

$$
Y_{3 / 2}^{(\text {direct })}=\left(\frac{90}{\pi^{2} g_{*}\left(T_{\mathrm{R}}\right)}\right)^{\frac{1}{4}} \frac{1}{32 \pi} \sqrt{\frac{m_{\Phi}}{X M_{\mathrm{G}}}} \times \begin{cases}16\left(\frac{m_{3 / 2}}{m_{\Phi}}\right)^{2} & \left(m_{z}^{2} \ll m_{\Phi} m_{3 / 2}\right) \\ \left(\frac{m_{z}}{m_{\Phi}}\right)^{4} & \left(3 m_{\Phi} m_{3 / 2} \ll m_{z}^{2} \ll m_{\Phi}^{2}\right) . \\ 1 & \left(m_{\Phi}^{2} \ll m_{z}^{2}\right)\end{cases}
$$

The gravitino yield from thermal bath is known to be [89-94]

$$
Y_{3 / 2}^{\text {(thermal })} \simeq \begin{cases}\min \left[2 \times 10^{-12}\left(1+\frac{m_{\tilde{\tilde{g}}^{2}}}{3 m_{3 / 2}^{2}}\right)\left(\frac{T_{\mathrm{R}}}{10^{10} \mathrm{GeV}}\right), \frac{0.42}{g_{* s\left(T_{3 / 2}\right)}}\right] & \left(T_{\mathrm{R}} \gtrsim m_{\mathrm{SUSY}}\right) \\ 0 & \left(T_{\mathrm{R}} \lesssim m_{\mathrm{SUSY}}\right)\end{cases}
$$

where $m_{\tilde{\mathrm{g}}}$ is the gaugino (gluino) mass at zero temperature, $m_{\mathrm{SUSY}}$ is the typical soft SUSY breaking mass. We take them as $m_{\mathrm{SUSY}}=m_{3 / 2}$, and $m_{\tilde{\mathrm{g}}}=2.8 \times 10^{-2} m_{3 / 2}$ (for $m_{3 / 2} \geq$ $10^{4.5} \mathrm{GeV}$; anomaly mediation) or $m_{\tilde{\mathrm{g}}}=m_{3 / 2}$ (for $m_{3 / 2}<10^{4.5} \mathrm{GeV}$; gravity mediation).

The inflaton decays into matter particles, gravitino, and SUSY breaking field. It also decays into other SUGRA sector particles $\left(T, S, \chi^{T}\right.$, and $\left.\chi^{S}\right)$ if kinematically possible, but the rate should be highly suppressed by the phase space factor. Even if the decay is possible, these particles decay shortly after they are produced if there are Giudice-Masiero terms $\left|J_{i j}\right| \sim \mathcal{O}(1)$. Moreover, gravitino abundance from decay of these SUGRA sector particles $X$ will be multiply suppressed by tiny branching ratios of $\operatorname{Br}\left(\Phi_{\mathrm{R} \pm} \rightarrow X+\right.$ anything $)$ and $\operatorname{Br}\left(X \rightarrow \psi_{3 / 2}+\right.$ anything $)$. Therefore we neglect effects of these SUGRA sector particles, and consider only the SUSY breaking field $z$ for the process of the type (iii).

The SUSY breaking field $z$ is produced as particles by the decay of inflaton, and it decays dominantly into a pair of gravitinos when $m_{\Phi}>2 m_{z} \gg m_{3 / 2}$ because the partial decay rate into them is enhanced by a factor $\left(m_{z} / m_{3 / 2}\right)^{2},[80,81]$

$$
\Gamma\left(z \rightarrow \psi_{3 / 2} \psi_{3 / 2}\right)=\frac{m_{z}^{5}}{96 \pi m_{3 / 2}^{2} M_{\mathrm{G}}^{2}},
$$

while partial decay rates of other channels are of order $\Gamma=\mathcal{O}\left(m_{z}^{3} / 4 \pi M_{\mathrm{G}}^{2}\right)$.

The gravitino yield as a decay product of particle $z$, which in turn is created by decay of the inflaton, leads to

$$
Y_{3 / 2}^{(\text {particle })}=\frac{2 n_{z}}{s}=\frac{3 T_{\mathrm{R}}}{m_{\Phi}} \operatorname{Br}\left(\Phi_{\mathrm{R} \pm} \rightarrow z z\right)=\frac{T_{\mathrm{R}} m_{z}^{2}}{16 \pi X m_{\Phi}^{3}} .
$$

Finally we consider the process of the type (iv). For matter fields, canonically normalized Hubble-induced mass is $\sqrt{2} H$. This value is close to that for critical damping $3 H / 2$, so matter fields rapidly moves to the instantaneous minimum, which can be regarded as zero 
for our purpose. The SUSY breaking field $z$ is also trapped near the origin until it decays at $H=H_{\mathrm{D}} \simeq \Gamma\left(z \rightarrow \psi_{3 / 2} \psi_{3 / 2}\right)$ or until it starts coherent oscillation at $H=H_{\mathrm{O}} \simeq m_{z}$. Here and hereafter the subscripts $\mathrm{R}, \mathrm{D}$, and $\mathrm{O}$ refer to the time of reheating, decay of $z$, and beginning of coherent oscillation of $z$, respectively. Assuming that the dominant channel is the model-independent anomaly-induced decay, $H_{\mathrm{R}} \simeq 2.2 \mathrm{GeV}$. For definiteness, we assume eqs. (2.11) and (2.12) for the SUSY breaking sector.

The VEV of $z$ is evaluated as $\langle z\rangle \simeq 2 \sqrt{3}\left(\frac{m_{3 / 2}}{m_{z}}\right)^{2}$, and the energy density of coherent oscillation $z$ is

$$
\rho_{z, \text { field }}=m_{z}^{2}\langle z\rangle^{2}=\frac{12 m_{3 / 2}^{4}}{m_{z}^{2}} \times\left\{\begin{array}{ll}
1 & \left(H>H_{\mathrm{O}}\right) \\
\left(\frac{a}{a_{\mathrm{O}}}\right)^{-3} & \left(H_{\mathrm{O}}>H\right)
\end{array},\right.
$$

where $a$ is the cosmic scale factor. The entropy density is

$$
s=\frac{4 \rho}{3 T}=\frac{4 H^{2}}{T} .
$$

The gravitino yield from coherent oscillation of $z$ is thus

$$
Y_{3 / 2}^{(\text {field })}=\frac{2 \rho_{z, \text { field }}}{m_{z} s}=\frac{6 m_{3 / 2}^{4} T_{\mathrm{R}}}{m_{z}^{5}} \quad\left(H_{\mathrm{O}}>H_{\mathrm{R}}>H_{\mathrm{D}}\right) .
$$

If the mass scale of $z$ is larger than the inflation scale, $2 m_{z}>m_{\Phi}, z$ goes close to its VEV during inflation, and the above quantity $Y_{3 / 2}^{(\text {field) }}$ is further suppressed by a factor $\left(m_{\Phi}^{2} / 2 m_{z}^{2}\right)$.

So far, we have implicitly assumed the decay of $z$ occurs at last. If the decay of $z$ occurs between $H_{\mathrm{O}}$ and $H_{\mathrm{R}}$, the energy density of gravitinos generated by the decay of coherent oscillation of $z$ at the time of reheating is

$$
\rho_{3 / 2}=\frac{12 m_{3 / 2}^{4}}{m_{z}^{2}}\left(\frac{a_{\mathrm{D}}}{a_{\mathrm{O}}}\right)^{-3}\left(\frac{a_{\mathrm{NR}}}{a_{\mathrm{D}}}\right)^{-4}\left(\frac{a_{\mathrm{R}}}{a_{\mathrm{NR}}}\right)^{-3},
$$

where NR stands for the time when gravitino becomes non-relativistic, $H_{\mathrm{NR}}=$ $\left(m_{3 / 2} / m_{z}\right)^{3 / 2} H_{D}$. The gravitino yield is

$$
Y_{3 / 2}^{(\text {field })}=\frac{6 m_{3 / 2}^{5} T_{\mathrm{R}}}{m_{z}^{6}} .
$$

If $z$ decays even earlier than $H_{\mathrm{O}}$, the gravitino energy density is given by

$$
\rho_{3 / 2}=\frac{12 m_{3 / 2}^{4}}{m_{z}^{2}}\left(\frac{a_{\mathrm{NR}}}{a_{\mathrm{D}}}\right)^{-4}\left(\frac{a_{\mathrm{R}}}{a_{\mathrm{NR}}}\right)^{-3},
$$

so the gravitino yield is

$$
Y_{3 / 2}^{(\text {field })}=\frac{6 m_{3 / 2}^{5} T_{\mathrm{R}}}{m_{z}^{4} H_{\mathrm{D}}^{2}} .
$$

In summary, the gravitino yield from coherent $z$ field is given by

$$
Y_{3 / 2}^{(\text {field })}= \begin{cases}\frac{6 m_{3 / 2}^{4} T_{\mathrm{R}}}{m_{z}^{5}} & \left(H_{\mathrm{O}}>H_{\mathrm{R}}>H_{\mathrm{D}}\right) \\ \frac{6 m_{3 / 2}^{5} T_{\mathrm{R}}}{m_{z}^{6}} & \left(H_{\mathrm{O}}>H_{\mathrm{D}}>H_{\mathrm{R}}\right) . \\ \frac{6 m_{3 / 2}^{5} T_{\mathrm{R}}}{m_{z}^{4} H_{\mathrm{D}}^{2}} & \left(H_{\mathrm{D}}>H_{\mathrm{O}}>H_{\mathrm{R}}\right)\end{cases}
$$




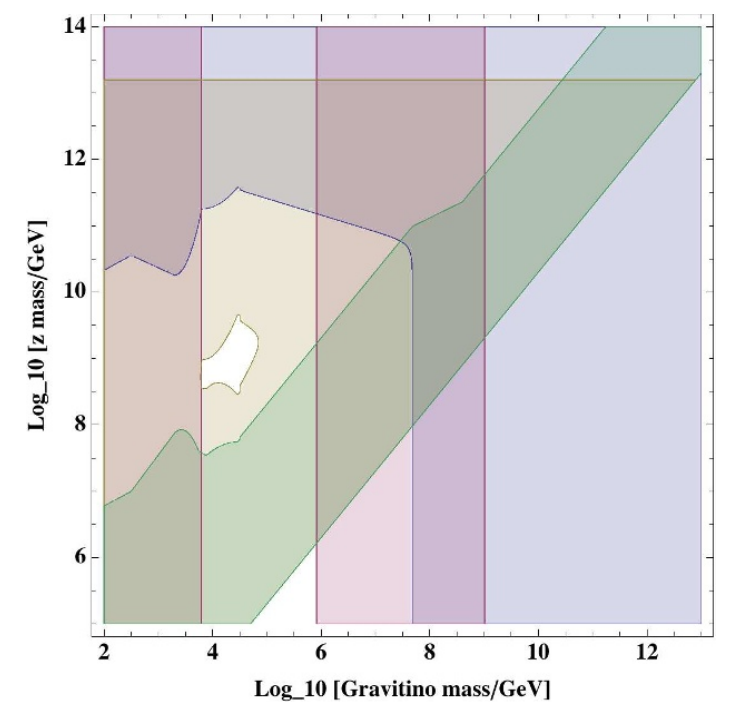

Figure 1. Constraint on masses of gravitino and SUSY breaking field from LSP overabundance from gravitino decay. Blue, red, yellow, and green shaded regions, corresponding to direct production, thermal production, $z$ particle decay, and $z$ coherent oscillation decay, are excluded.

Because we assume no entropy production after inflaton decay until gravitino decay, the denominators of every $Y_{3 / 2}$ are common, so the cosmologically relevant gravitino yield is the sum of all four terms, $Y_{3 / 2}^{\text {(total) }}=Y_{3 / 2}^{(\text {direct })}+Y_{3 / 2}^{(\text {thermal })}+Y_{3 / 2}^{(\text {particle })}+Y_{3 / 2}^{(\text {field })}$.

Now that we have derived generic expressions for gravitino abundance, let us discuss its cosmological consequences for a minimal setup. Gravitinos heavier than about $30 \mathrm{TeV}$ decay before big bang nucleosynthesis (BBN), but lightest supersymmetric particles (LSPs) produced by the gravitino decay chain may exceed the observed dark matter abundance. Such a constraint is shown in figure 1 assuming wino LSP of anomaly mediation for $m_{3 / 2} \geq$ $10^{4.5} \mathrm{GeV}$. For smaller gravitino mass, $m_{3 / 2}<10^{4.5} \mathrm{GeV}$, gravitino decay affects light element abundance. We assume gravity mediation for this mass region and impose the standard BBN constraints [94] on the parameter space in figure 1. In this figure, the dominant decay mode of the inflaton is assumed to be a model-independent one, namely the anomaly-induced decay into gauge bosons and gauginos as discussed in subsection 3.1.3. The inflaton mass is taken as $m_{\Phi}=3.2 \times 10^{13} \mathrm{GeV}$, and the reheating temperature after inflaton decay is $T_{\mathrm{R}} \simeq 1.0 \times 10^{9} \mathrm{GeV}$. Instantaneous reheating occurs in spite of the Plancksuppressed interaction [95]. As can be seen from the figure, most of the parameter space are excluded. The lower unshaded region is also excluded by the standard constraint of the cosmological moduli problem [96-98] unless baryon asymmetry is regenerated e.g. by the Affleck-Dine mechanism [99]. (In this case the modulus (Polonyi) field is the SUSY breaking field $z$.) Note that the range of gravitino mass $10^{6} \mathrm{GeV} \lesssim m_{3 / 2} \lesssim 3 \times 10^{11} \mathrm{GeV}$ (corresponding to $3 \mathrm{TeV} \lesssim m_{\text {wino }} \lesssim T_{\mathrm{R}}$; not shown in the figure) is excluded by thermally produced wino abundance [100] even without considering the wino LSP from gravitino decay. See also ref. [101] for non-thermal production of wino dark matter via the decay of long-lived particles. As usual, this problem is ameliorated or solved by assuming $R$-parity breaking so that LSP decays or thermal inflation [102] so that it is diluted. 


\section{Summary and discussion}

In this paper, we studied coupling of the SUSY Starobinsky model to matter sector in the old-minimal supergravity, inflaton decay and its cosmological consequences. To this end, we first transformed the supergravity theory of supercurvature $\mathcal{R}$ minimally coupled to matter to an equivalent one in the form of the standard no-scale type supergravity of inflaton $T$ plus another matter superfield $S$. The notable feature there is that the interactions of the inflaton $T$ to other superfields in the theory are completely determined by the fact that the origin of $T$ is a Lagrange multiplier. In particular, the inflaton $T$ does not enter in the gauge kinetic function. These are characteristic features of the SUSY Starobinsky model, unlike some other SUGRA models having Starobinsky-like scalar potentials.

On the other hand, interactions of $S$ have more freedom. In this paper, we assumed minimal coupling between SUGRA sector and matter sector in the first place, but it is not protected by any symmetries so more general coupling between $S$ and matter are possible. It may enhance decay rates of inflaton into matter through mixing between $T$ and $S$, which results in a suppressed branching ratio into gravitino.

We focused on model-independent decay channel of inflaton into gauge sector via the anomaly-induced decay in section 4 , but presence of heavy matter, like right-handed (s)neutrinos, and large quadratic holomorphic term $J_{i j}$ in Kähler potential, which is used for the Giudice-Masiero mechanism [103], are helpful to reheat the universe efficiently. These are simply because there are decay modes whose rates are proportional to matter mass or $J_{i j}$.

Taking anomaly-induced decay into the gauge sector as the dominant decay channel, the lower limit of the reheating temperature is a similar value, $T_{\mathrm{R}} \simeq 1.0 \times 10^{9} \mathrm{GeV}$, to that of the non-SUSY original Starobinsky model, and it is consistent with thermal leptogenesis [104]. The most striking difference to the non-SUSY case is presence of the built-in long lived particle in the theory, gravitino. We assumed gravity/anomaly mediation of SUSY breaking, and estimated the amount of LSPs produced from decay of gravitino, which is produced either by direct decay of inflaton, thermal scattering, decay of SUSY breaking particle or field $z$. The result is that most of the parameter space $\left(m_{3 / 2}, m_{z}\right)$ is excluded unless $R$-parity is broken or thermal inflation occurs. Thus, our prediction of the mass of gravitino is $10^{4} \mathrm{GeV} \lesssim m_{3 / 2} \lesssim 10^{5} \mathrm{GeV}$. A way around this is considering more general coupling between SUGRA sector and matter sector in the original higher supercurvature SUGRA theory.

\section{Acknowledgments}

We would like to thank Iannis Dalianis for clarifying the model in ref. [70]. TT thanks Motoi Endo, Kazunori Nakayama, Fuminobu Takahashi, and Masahiro Takimoto for valuable discussion. TT was supported partly by a grant of Advanced Leading Graduate Course for Photon Science in the University of Tokyo, and partly by a Grant-in-Aid for JSPS Fellows, and a Grant-in-Aid of the JSPS under No. 26-10619. YW acknowledges supports from the JSPS Research Fellowship for Young Scientists No. 269337 and the Munich Institute 
for Astro- and Particle Physics (MIAPP) of the DFG cluster of excellence "Origin and Structure of the Universe." YY acknowledges support from the JSPS Research Fellowship for Young Scientists No. 264236. JY acknowledges support form the JSPS Grant-in-Aid for Scientific Research (B) No. 23340058.

\section{A Duality transformation of higher derivative SUGRA models}

In this appendix, we briefly review the duality transformation between the higher derivative SUGRA system and the standard one described in section 2. We explicitly show that the superpotential and the gauge kinetic function in the standard SUGRA are linear in and independent of $T$, respectively. We also discuss some generalizations.

The action including general couplings between $\mathcal{R}$ and matters [38] is given by

$$
\begin{aligned}
S= & \int d^{4} x d^{4} \theta \operatorname{EN}\left(\mathcal{R}, \overline{\mathcal{R}}, \phi, \bar{\phi} e^{g V}\right) \\
& +\left[\int d^{4} x d^{2} \Theta 2 \mathscr{E}\left(F(\mathcal{R}, \phi)+\frac{1}{4} h_{A B}(\mathcal{R}, \phi) W^{A} W^{B}\right)+\text { H.c. }\right],
\end{aligned}
$$

where $\mathcal{R}, \phi$ and $W^{A}$ are the same as in section $2, N$ is a real function of $\mathcal{R}, \phi$ and their conjugates, and $F$ and $h_{A B}$ are holomorphic functions of $\mathcal{R}$ and $\phi$. By introducing the Lagrange multiplier chiral multiplet $T$ and a chiral multiplet $S$, the action (A.1) becomes the following form,

$$
\begin{aligned}
S= & \int d^{4} x d^{4} \theta E N\left(S, \bar{S}, \phi, \bar{\phi} e^{g V}\right) \\
& +\left[\int d^{4} x d^{2} \Theta 2 \mathscr{E}\left(2 T(S-\mathcal{R})+F(S, \phi)+\frac{1}{4} h_{A B}(S, \phi) W^{A} W^{B}\right)+\text { H.c. }\right] .
\end{aligned}
$$

Varying it with respect to $T$ yields the equation $\mathcal{R}=S$, and we obtain the original action (A.1). We can also rewrite the action (A.2) into the standard SUGRA form not containing higher curvature terms as

$$
\begin{aligned}
S= & \int d^{4} x d^{4} \theta E\left[N\left(S, \bar{S}, \phi, \bar{\phi} e^{g V}\right)-(T+\bar{T})\right] \\
& +\left[\int d^{4} x d^{2} \Theta 2 \mathscr{E}\left(2 T S+F(S, \phi)+\frac{1}{4} h_{A B}(S, \phi) W^{A} W^{B}\right)+\text { H.c. }\right] \\
= & \int d^{4} x d^{2} \Theta 2 \mathscr{E}\left[\frac{3}{8}(\overline{\mathscr{D}} \overline{\mathscr{D}}-8 \mathcal{R}) e^{-K / 3}+W+\frac{1}{4} h_{A B}(S, \phi) W^{A} W^{B}\right]+\text { H.c. }
\end{aligned}
$$

where

$$
\begin{aligned}
K & =-3 \ln \left(\frac{T+\bar{T}-N\left(S, \bar{S}, \phi, \bar{\phi} e^{g V}\right)}{3}\right), \\
W & =2 T S+F(S, \phi) .
\end{aligned}
$$

Notice that in the dual action (A.3), $T$ does not appear in the gauge kinetic function $h_{A B}$ even if there are couplings between $\mathcal{R}$ and $W_{\alpha}^{A}$ in the original action (A.1). The absence of 
$T$ in the gauge kinetic function $h_{A B}$ is a remarkable feature of the Starobinsky inflation in old-minimal SUGRA models, which restricts the main reheating processes to the anomaly induced decays into the gauge sector as discussed in section 3 .

We have shown that the naive generalization of the action (2.1) does not contain $T$ dependence in gauge kinetic function $h_{A B}$. One may wonder what happens if we introduce dependence of the gauge kinetic function on $T$ in eq. (A.3) and transform it back to a higher derivative SUGRA. As a minimal extension of eq. (A.3), let us consider the following action in which the gauge kinetic function linearly depends on $T$,

$$
\begin{aligned}
S= & \int d^{4} x d^{4} \theta E\left[N\left(S, \bar{S}, \phi, \bar{\phi} e^{g V}\right)-(T+\bar{T})\right] \\
& +\left[\int d^{4} x d^{2} \Theta 2 \mathscr{E}\left(2 T S+F(S, \phi)+\left(\frac{1}{4} h_{A B}(S, \phi)-2 H_{A B} T\right) W^{A} W^{B}\right)+\text { H.c. }\right],
\end{aligned}
$$

where $H_{A B}$ is a constant. Here, to obtain the dual action of (A.6), we follow the way discussed in ref. [67]. We can recast the action (A.6) into the dual form as

$$
\begin{aligned}
S= & \int d^{4} x d^{4} \theta E N\left(S, \bar{S}, \phi, \bar{\phi} e^{g V}\right) \\
& +\left[\int d^{4} x d^{2} \Theta 2 \mathscr{E}\left(2 T\left(S-\mathcal{R}-H_{A B} W^{A} W^{B}\right)+F(S, \phi)+\frac{1}{4} h_{A B}(S, \phi) W^{A} W^{B}\right)+\text { H.c. }\right] .
\end{aligned}
$$

Varying the above action with respect to $T$ yields $S=\mathcal{R}+H_{A B} W^{A} W^{B}$. Substituting it into the action gives

$$
\begin{aligned}
S= & \int d^{4} x d^{4} \theta E N\left(\mathcal{R}+H_{A B} W^{A} W^{B}, \overline{\mathcal{R}}+\bar{H}_{A B} \bar{W}^{A} \bar{W}^{B}, \phi, \bar{\phi} e^{g V}\right) \\
& +\left[\int d^{4} x d^{2} \Theta 2 \mathscr{E}\left(F\left(\mathcal{R}+H_{A B} W^{A} W^{B}, \phi\right)+\frac{1}{4} h_{A B}\left(\mathcal{R}+H_{C D} W^{C} W^{D}, \phi\right) W^{A} W^{B}\right)+\text { H.c. }\right] .
\end{aligned}
$$

Notice that the dual action (A.8) contains higher dimensional operators involving $H_{A B} W^{A} W^{B}$. It means that the theory contains the higher derivative terms of the gauge multiplets $V^{A}$. In such a case, the inflaton $T$ can decay into gauge bosons and gauginos through tree level couplings in the gauge kinetic function (or in the Kähler potential depending on the shift of $S$ ). Then, reheating processes can be different from the ones we discussed in section 3.

For completeness, we finally discuss a possibility that the superpotential and the gauge kinetic function are non-linear in $T$. We generalize the $F$-term action as

$$
\begin{aligned}
S= & \int d^{4} x d^{4} \theta E\left[N\left(S, \bar{S}, \phi, \bar{\phi} e^{g V}\right)-(T+\bar{T})\right] \\
& +\left[\int d^{4} x d^{2} \Theta 2 \mathscr{E}\left(F(T, S, \phi)+\frac{1}{4} h_{A B}(T, S, \phi) W^{A} W^{B}\right)+\text { H.c. }\right],
\end{aligned}
$$

where $F(T, S, \phi)$ and $h_{A B}(T, S, \phi)$ are holomorphic functions of $T, S$, and $\phi^{i}$. We can rewrite the action (A.9) as

$$
\begin{aligned}
S= & \int d^{4} x d^{4} \theta E\left(N\left(S, \bar{S}, \phi, \bar{\phi} e^{g V}\right)\right) \\
& +\left[\int d^{4} x d^{2} \Theta 2 \mathscr{E}\left(-2 \mathcal{R}+F(T, S, \phi)+\frac{1}{4} h_{A B}(T, S, \phi) W^{A} W^{B}\right)+\text { H.c. }\right] .
\end{aligned}
$$


Varying the above action with respect to $T$ yields

$$
F_{T}(T, S, \phi)+\frac{1}{4} h_{A B, T}(T, S, \phi) W^{A} W^{B}-2 \mathcal{R}=0,
$$

and it can be implicitly solved as $S=S(\mathcal{R}, T, \phi)$. Substituting it to eq. (A.9) leads to a higher derivative SUGRA depending on $\mathcal{R}, \phi^{i}$, and an additional matter $T$. Notice that dependence on the additional matter $T$ vanishes if and only if $F(T, S, \phi)$ and $h_{A B}(T, S, \phi)$ are linear functions of $T$. Therefore, the non-linear dependence of $T$ in the superpotential or the gauge kinetic function requires a new chiral multiplet $T$ in the dual higher derivative SUGRA theory.

In this paper, we focus on the case that $T$ appears as the degree of freedom purely originated from the higher derivative SUGRA terms. In this case, the corresponding action of the standard SUGRA is given by eq. (2.3) [or more generally by eq. (A.3)].

Open Access. This article is distributed under the terms of the Creative Commons Attribution License (CC-BY 4.0), which permits any use, distribution and reproduction in any medium, provided the original author(s) and source are credited.

\section{References}

[1] WMAP collaboration, G. Hinshaw et al., Nine-year Wilkinson Microwave Anisotropy Probe (WMAP) observations: cosmological parameter results, Astrophys. J. Suppl. 208 (2013) 19 [arXiv:1212.5226] [INSPIRE].

[2] Planck collaboration, P.A.R. Ade et al., Planck 2013 results. XVI. Cosmological parameters, Astron. Astrophys. 571 (2014) A16 [arXiv:1303.5076] [InSPIRE].

[3] Planck collaboration, P.A.R. Ade et al., Planck 2013 results. XXII. Constraints on inflation, Astron. Astrophys. 571 (2014) A22 [arXiv:1303.5082] [InSPIRE].

[4] A.A. Starobinsky, A new type of isotropic cosmological models without singularity, Phys. Lett. B 91 (1980) 99 [INSPIRE].

[5] A.A. Starobinsky, Nonsingular model of the universe with the quantum gravitational de Sitter stage and its observational consequences, in Proc. of the Second Seminar "Quantum Theory of Gravity", Moscow Russia October 13-15 1981, INR Press, Moscow Russia (1982), pg. 58 [INSPIRE].

[6] K. Sato, First order phase transition of a vacuum and expansion of the universe, Mon. Not. Roy. Astron. Soc. 195 (1981) 467 [INSPIRE].

[7] A.H. Guth, The inflationary universe: a possible solution to the horizon and flatness problems, Phys. Rev. D 23 (1981) 347 [INSPIRE].

[8] A.D. Linde, A new inflationary universe scenario: a possible solution of the horizon, flatness, homogeneity, isotropy and primordial monopole problems, Phys. Lett. B 108 (1982) 389 [INSPIRE].

[9] A. Albrecht and P.J. Steinhardt, Cosmology for grand unified theories with radiatively induced symmetry breaking, Phys. Rev. Lett. 48 (1982) 1220 [INSPIRE].

[10] A.D. Linde, Chaotic inflation, Phys. Lett. B 129 (1983) 177 [INSPIRE]. 
[11] J. Yokoyama, Inflation: 1980-201X, Prog. Theor. Exp. Phys. 2014 (2014) 06B103 [INSPIRE].

[12] S. Tsujikawa, Distinguishing between inflationary models from cosmic microwave background, Prog. Theor. Exp. Phys. 2014 (2014) 06B104 [arXiv:1401.4688] [INSPIRE].

[13] M.B. Mijic, M.S. Morris and W.-M. Suen, The $R^{2}$ cosmology: inflation without a phase transition, Phys. Rev. D 34 (1986) 2934 [INSPIRE].

[14] B. Whitt, Fourth order gravity as general relativity plus matter, Phys. Lett. B 145 (1984) 176 [INSPIRE].

[15] K.-I. Maeda, Inflation as a transient attractor in $R^{2}$ cosmology, Phys. Rev. D 37 (1988) 858 [inSPIRE].

[16] A. Vilenkin, Classical and quantum cosmology of the Starobinsky inflationary model, Phys. Rev. D 32 (1985) 2511 [inSPIRE].

[17] Y. Watanabe and E. Komatsu, Reheating of the universe after inflation with $f(\phi) R$ gravity, Phys. Rev. D 75 (2007) 061301 [gr-qc/0612120] [INSPIRE].

[18] T. Faulkner, M. Tegmark, E.F. Bunn and Y. Mao, Constraining $f(R)$ gravity as a scalar tensor theory, Phys. Rev. D 76 (2007) 063505 [astro-ph/0612569] [INSPIRE].

[19] D.S. Gorbunov and A.G. Panin, Scalaron the mighty: producing dark matter and baryon asymmetry at reheating, Phys. Lett. B 700 (2011) 157 [arXiv: 1009.2448] [INSPIRE].

[20] E.V. Arbuzova, A.D. Dolgov and L. Reverberi, Cosmological evolution in $R^{2}$ gravity, JCAP 02 (2012) 049 [arXiv: 1112.4995] [INSPIRE].

[21] H. Motohashi and A. Nishizawa, Reheating after $f(R)$ inflation, Phys. Rev. D 86 (2012) 083514 [arXiv:1204.1472] [InSPIRE].

[22] I. Rudenok, Y. Shtanov and S. Vilchinskii, Post-inflationary preheating with weak coupling, Phys. Lett. B 733 (2014) 193 [arXiv:1401.7298] [INSPIRE].

[23] N. Takeda and Y. Watanabe, No quasistable scalaron lump forms after $R^{2}$ inflation, Phys. Rev. D 90 (2014) 023519 [arXiv:1405.3830] [INSPIRE].

[24] V.F. Mukhanov and G.V. Chibisov, Quantum fluctuation and nonsingular universe (in Russian), JETP Lett. 33 (1981) 532 [Pisma Zh. Eksp. Teor. Fiz. 33 (1981) 549] [InSPIRE].

[25] V.F. Mukhanov, CMB, quantum fluctuations and the predictive power of inflation, astro-ph/0303077 [INSPIRE].

[26] BICEP2 collaboration, P.A.R. Ade et al., Detection of B-mode polarization at degree angular scales by BICEP2, Phys. Rev. Lett. 112 (2014) 241101 [arXiv:1403.3985] [INSPIRE].

[27] M.J. Mortonson and U. Seljak, A joint analysis of Planck and BICEP2 B modes including dust polarization uncertainty, JCAP 10 (2014) 035 [arXiv: 1405.5857] [INSPIRE].

[28] R. Flauger, J.C. Hill and D.N. Spergel, Toward an understanding of foreground emission in the BICEP2 region, JCAP 08 (2014) 039 [arXiv:1405.7351] [INSPIRE].

[29] Planck collaboration, R. Adam et al., Planck intermediate results. XXX. The angular power spectrum of polarized dust emission at intermediate and high galactic latitudes, arXiv: 1409.5738 [INSPIRE]. 
[30] D.Z. Freedman, P. van Nieuwenhuizen and S. Ferrara, Progress toward a theory of supergravity, Phys. Rev. D 13 (1976) 3214 [InSPIRE].

[31] S. Deser and B. Zumino, Consistent supergravity, Phys. Lett. B 62 (1976) 335 [InSPIRE].

[32] S. Ferrara and P. van Nieuwenhuizen, The auxiliary fields of supergravity, Phys. Lett. B 74 (1978) 333 [inSPIRE].

[33] K.S. Stelle and P.C. West, Minimal auxiliary fields for supergravity, Phys. Lett. B 74 (1978) 330 [inSPIRE].

[34] E.S. Fradkin and M.A. Vasiliev, $S$ matrix for theories that admit closure of the algebra with the aid of auxiliary fields: the auxiliary fields in supergravity, Lett. Nuovo Cim. 22 (1978) 651 [INSPIRE].

[35] M.F. Sohnius and P.C. West, An alternative minimal off-shell version of $N=1$ supergravity, Phys. Lett. B 105 (1981) 353 [INSPIRE].

[36] S. Ferrara, L. Girardello, T. Kugo and A. Van Proeyen, Relation between different auxiliary field formulations of $N=1$ supergravity coupled to matter, Nucl. Phys. B 223 (1983) 191 [INSPIRE].

[37] S. Ferrara, M.T. Grisaru and P. van Nieuwenhuizen, Poincaré and conformal supergravity models with closed algebras, Nucl. Phys. B 138 (1978) 430 [INSPIRE].

[38] S. Cecotti, Higher derivative supergravity is equivalent to standard supergravity coupled to matter. 1, Phys. Lett. B 190 (1987) 86 [INSPIRE].

[39] R. Kallosh and A. Linde, Superconformal generalizations of the Starobinsky model, JCAP 06 (2013) 028 [arXiv: 1306.3214] [INSPIRE].

[40] A. Hindawi, B.A. Ovrut and D. Waldram, Four-dimensional higher derivative supergravity and spontaneous supersymmetry breaking, Nucl. Phys. B 476 (1996) 175 [hep-th/9511223] [INSPIRE].

[41] S.V. Ketov, On the supersymmetrization of inflation in $f(R)$ gravity, Prog. Theor. Exp. Phys. 2013 (2013) 123B04 [arXiv:1309.0293] [INSPIRE].

[42] S.V. Ketov and T. Terada, Old-minimal supergravity models of inflation, JHEP 12 (2013) 040 [arXiv:1309.7494] [INSPIRE].

[43] S.J. Gates Jr. and S.V. Ketov, Superstring-inspired supergravity as the universal source of inflation and quintessence, Phys. Lett. B 674 (2009) 59 [arXiv:0901.2467] [INSPIRE].

[44] S.V. Ketov, Supergravity and early universe: the meeting point of cosmology and high-energy physics, Int. J. Mod. Phys. A 28 (2013) 1330021 [arXiv:1201.2239] [InSPIRE].

[45] S.V. Ketov, Chaotic inflation in F(R) supergravity, Phys. Lett. B 692 (2010) 272 [arXiv: 1005.3630] [INSPIRE].

[46] S.V. Ketov and A.A. Starobinsky, Inflation and non-minimal scalar-curvature coupling in gravity and supergravity, JCAP 08 (2012) 022 [arXiv: 1203.0805] [INSPIRE].

[47] S.V. Ketov and T. Terada, New actions for modified gravity and supergravity, JHEP 07 (2013) 127 [arXiv:1304.4319] [INSPIRE].

[48] S.V. Ketov and S. Tsujikawa, Consistency of inflation and preheating in $F(R)$ supergravity, Phys. Rev. D 86 (2012) 023529 [arXiv: 1205. 2918] [INSPIRE]. 
[49] Y. Watanabe and J. Yokoyama, Gravitational modulated reheating and non-Gaussianity in supergravity $R^{2}$ inflation, Phys. Rev. D 87 (2013) 103524 [arXiv:1303.5191] [INSPIRE].

[50] J. Ellis, D.V. Nanopoulos and K.A. Olive, No-scale supergravity realization of the Starobinsky model of inflation, Phys. Rev. Lett. 111 (2013) 111301 [arXiv:1305.1247] [INSPIRE].

[51] J. Ellis, D.V. Nanopoulos and K.A. Olive, Starobinsky-like inflationary models as avatars of no-scale supergravity, JCAP 10 (2013) 009 [arXiv:1307.3537] [INSPIRE].

[52] S. Ferrara, R. Kallosh and A. Van Proeyen, On the supersymmetric completion of $R+R^{2}$ gravity and cosmology, JHEP 11 (2013) 134 [arXiv:1309.4052] [INSPIRE].

[53] S. Ferrara, A. Kehagias and M. Porrati, Vacuum structure in a chiral $R+R^{n}$ modification of pure supergravity, Phys. Lett. B 727 (2013) 314 [arXiv:1310.0399] [INSPIRE].

[54] S. Cecotti, S. Ferrara, M. Porrati and S. Sabharwal, New minimal higher derivative supergravity coupled to matter, Nucl. Phys. B 306 (1988) 160 [INSPIRE].

[55] F. Farakos, A. Kehagias and A. Riotto, On the Starobinsky model of inflation from supergravity, Nucl. Phys. B 876 (2013) 187 [arXiv:1307.1137] [INSPIRE].

[56] S. Ferrara, R. Kallosh, A. Linde and M. Porrati, Minimal supergravity models of inflation, Phys. Rev. D 88 (2013) 085038 [arXiv:1307.7696] [INSPIRE].

[57] S. Ferrara, R. Kallosh, A. Linde and M. Porrati, Higher order corrections in minimal supergravity models of inflation, JCAP 11 (2013) 046 [arXiv:1309.1085] [INSPIRE].

[58] W. Buchmüller, V. Domcke and K. Schmitz, Superconformal D-term inflation, JCAP 04 (2013) 019 [arXiv:1210.4105] [INSPIRE].

[59] W. Buchmüller, V. Domcke and K. Kamada, The Starobinsky model from superconformal D-term inflation, Phys. Lett. B 726 (2013) 467 [arXiv: 1306.3471] [INSPIRE].

[60] J. Alexandre, N. Houston and N.E. Mavromatos, Starobinsky-type inflation in dynamical supergravity breaking scenarios, Phys. Rev. D 89 (2014) 027703 [arXiv:1312.5197] [INSPIRE].

[61] C. Pallis, Linking Starobinsky-type inflation in no-scale supergravity to MSSM, JCAP 04 (2014) 024 [arXiv: 1312.3623] [INSPIRE].

[62] A. Hindawi, B.A. Ovrut and D. Waldram, Soft supersymmetry breaking induced by higher derivative supergravitation in the electroweak standard model, Phys. Lett. B 381 (1996) 154 [hep-th/9602075] [INSPIRE].

[63] S. Ferrara and M. Porrati, Minimal $R+R^{2}$ supergravity models of inflation coupled to matter, Phys. Lett. B 737 (2014) 135 [arXiv:1407.6164] [INSPIRE].

[64] K. Kamada and J. Yokoyama, Topological inflation from the Starobinsky model in supergravity, Phys. Rev. D 90 (2014) 103520 [arXiv:1405.6732] [InSPIRE].

[65] J. Wess and J. Bagger, Supersymmetry and supergravity, Princeton Univ. Pr., Princeton U.S.A. (1992) [INSPIRE].

[66] G.A. Diamandis et al., Inflation in $R^{2}$ supergravity with non-minimal superpotentials, arXiv: 1411.5785 [INSPIRE].

[67] S. Cecotti and R. Kallosh, Cosmological attractor models and higher curvature supergravity, JHEP 05 (2014) 114 [arXiv: 1403.2932] [INSPIRE]. 
[68] T. Kugo and S. Uehara, Improved superconformal gauge conditions in the $N=1$ supergravity Yang-Mills matter system, Nucl. Phys. B 222 (1983) 125 [INSPIRE].

[69] H. Abe, S. Aoki, F. Hasegawa and Y. Yamada, Illustrating SUSY breaking effects on various inflation mechanisms, JHEP 01 (2015) 026 [arXiv: 1408.4875] [INSPIRE].

[70] I. Dalianis, F. Farakos, A. Kehagias, A. Riotto and R. von Unge, Supersymmetry breaking and inflation from higher curvature supergravity, JHEP 01 (2015) 043 [arXiv:1409.8299] [INSPIRE].

[71] G.F. Giudice and A. Strumia, Probing high-scale and split supersymmetry with Higgs mass measurements, Nucl. Phys. B 858 (2012) 63 [arXiv:1108.6077] [InSPIRE].

[72] M. Endo, F. Takahashi and T.T. Yanagida, Inflaton decay in supergravity, Phys. Rev. D 76 (2007) 083509 [arXiv:0706.0986] [INSPIRE].

[73] M. Endo, K. Kadota, K.A. Olive, F. Takahashi and T.T. Yanagida, The decay of the inflaton in no-scale supergravity, JCAP 02 (2007) 018 [hep-ph/0612263] [INSPIRE].

[74] M. Endo, F. Takahashi and T.T. Yanagida, Anomaly-induced inflaton decay and gravitino-overproduction problem, Phys. Lett. B 658 (2008) 236 [hep-ph/0701042] [INSPIRE].

[75] Y. Watanabe, Rate of gravitational inflaton decay via gauge trace anomaly, Phys. Rev. D 83 (2011) 043511 [arXiv:1011.3348] [INSPIRE].

[76] D. Gorbunov and A. Tokareva, $R^{2}$-inflation with conformal SM Higgs field, JCAP 12 (2013) 021 [arXiv:1212.4466] [INSPIRE].

[77] M. Dine, R. Kitano, A. Morisse and Y. Shirman, Moduli decays and gravitinos, Phys. Rev. D 73 (2006) 123518 [hep-ph/0604140] [INSPIRE].

[78] M. Endo, K. Hamaguchi and F. Takahashi, Moduli/inflaton mixing with supersymmetry breaking field, Phys. Rev. D 74 (2006) 023531 [hep-ph/0605091] [INSPIRE].

[79] W. Buchmüller, K. Hamaguchi, M. Ratz and T. Yanagida, Supergravity at colliders, Phys. Lett. B 588 (2004) 90 [hep-ph/0402179] [INSPIRE].

[80] M. Endo, K. Hamaguchi and F. Takahashi, Moduli-induced gravitino problem, Phys. Rev. Lett. 96 (2006) 211301 [hep-ph/0602061] [INSPIRE].

[81] S. Nakamura and M. Yamaguchi, Gravitino production from heavy moduli decay and cosmological moduli problem revived, Phys. Lett. B 638 (2006) 389 [hep-ph/0602081] [INSPIRE].

[82] M. Endo, K. Hamaguchi and T. Terada, Scalar decay into gravitinos in the presence of D-term SUSY breaking, Phys. Rev. D 86 (2012) 083543 [arXiv:1208.4432] [INSPIRE].

[83] M. Kawasaki, F. Takahashi and T.T. Yanagida, Gravitino overproduction in inflaton decay, Phys. Lett. B 638 (2006) 8 [hep-ph/0603265] [INSPIRE].

[84] M. Kawasaki, F. Takahashi and T.T. Yanagida, The gravitino-overproduction problem in inflationary universe, Phys. Rev. D 74 (2006) 043519 [hep-ph/0605297] [INSPIRE].

[85] M. Endo, M. Kawasaki, F. Takahashi and T.T. Yanagida, Inflaton decay through supergravity effects, Phys. Lett. B 642 (2006) 518 [hep-ph/0607170] [INSPIRE].

[86] T. Asaka, S. Nakamura and M. Yamaguchi, Gravitinos from heavy scalar decay, Phys. Rev. D 74 (2006) 023520 [hep-ph/0604132] [INSPIRE]. 
[87] K. Nakayama, F. Takahashi and T.T. Yanagida, Eluding the gravitino overproduction in inflaton decay, Phys. Lett. B 718 (2012) 526 [arXiv:1209.2583] [INSPIRE].

[88] J.L. Evans, M.A.G. Garcia and K.A. Olive, The moduli and gravitino (non)-problems in models with strongly stabilized moduli, JCAP 03 (2014) 022 [arXiv:1311.0052] [INSPIRE].

[89] K. Nakayama, F. Takahashi and T.T. Yanagida, Gravitino problem in supergravity chaotic inflation and SUSY breaking scale after BICEP2, Phys. Lett. B 734 (2014) 358 [arXiv: 1404.2472] [INSPIRE].

[90] M. Bolz, A. Brandenburg and W. Buchmüller, Thermal production of gravitinos, Nucl. Phys. B 606 (2001) 518 [Erratum ibid. B 790 (2008) 336] [hep-ph/0012052] [INSPIRE].

[91] J. Pradler and F.D. Steffen, Thermal gravitino production and collider tests of leptogenesis, Phys. Rev. D 75 (2007) 023509 [hep-ph/0608344] [INSPIRE].

[92] J. Pradler and F.D. Steffen, Constraints on the reheating temperature in gravitino dark matter scenarios, Phys. Lett. B 648 (2007) 224 [hep-ph/0612291] [INSPIRE].

[93] V.S. Rychkov and A. Strumia, Thermal production of gravitinos, Phys. Rev. D 75 (2007) 075011 [hep-ph/0701104] [INSPIRE].

[94] K. Kohri, T. Moroi and A. Yotsuyanagi, Big-bang nucleosynthesis with unstable gravitino and upper bound on the reheating temperature, Phys. Rev. D 73 (2006) 123511 [hep-ph/0507245] [INSPIRE].

[95] K. Harigaya and K. Mukaida, Thermalization after/during reheating, JHEP 05 (2014) 006 [arXiv: 1312.3097] [INSPIRE].

[96] G.D. Coughlan, W. Fischler, E.W. Kolb, S. Raby and G.G. Ross, Cosmological problems for the Polonyi potential, Phys. Lett. B 131 (1983) 59 [InSPIRE].

[97] T. Banks, D.B. Kaplan and A.E. Nelson, Cosmological implications of dynamical supersymmetry breaking, Phys. Rev. D 49 (1994) 779 [hep-ph/9308292] [INSPIRE].

[98] B. de Carlos, J.A. Casas, F. Quevedo and E. Roulet, Model independent properties and cosmological implications of the dilaton and moduli sectors of $4 D$ strings, Phys. Lett. B 318 (1993) 447 [hep-ph/9308325] [INSPIRE].

[99] I. Affleck and M. Dine, A new mechanism for baryogenesis, Nucl. Phys. B 249 (1985) 361 [INSPIRE].

[100] J. Hisano, S. Matsumoto, M. Nagai, O. Saito and M. Senami, Non-perturbative effect on thermal relic abundance of dark matter, Phys. Lett. B 646 (2007) 34 [hep-ph/0610249] [INSPIRE].

[101] T. Moroi, M. Nagai and M. Takimoto, Non-thermal production of wino dark matter via the decay of long-lived particles, JHEP 07 (2013) 066 [arXiv:1303.0948] [INSPIRE].

[102] D.H. Lyth and E.D. Stewart, Thermal inflation and the moduli problem, Phys. Rev. D 53 (1996) 1784 [hep-ph/9510204] [INSPIRE].

[103] G.F. Giudice and A. Masiero, A natural solution to the $\mu$ problem in supergravity theories, Phys. Lett. B 206 (1988) 480 [INSPIRE].

[104] M. Fukugita and T. Yanagida, Baryogenesis without grand unification, Phys. Lett. B 174 (1986) 45 [INSPIRE]. 